JOURNAL OF INTEGRAL EQUATIONS

AND APPLICATIONS

Volume 16, Number 4, Winter 2004

\title{
THE FINITE LAPLACE TRANSFORM FOR SOLVING A WEAKLY SINGULAR INTEGRAL EQUATION OCCURRING IN TRANSFER THEORY
}

\author{
B. RUTILY AND L. CHEVALLIER
}

\begin{abstract}
We solve a weakly singular integral equation by Laplace transformation over a finite interval of $\mathbf{R}$ The equation is transformed into a Cauchy integral equation, whose resolution amounts to solving two Fredholm integral equations of the second kind with regular kernels. This classical scheme is used to clarify the emergence of the auxiliary functions expressing the solution of the problem. There are four such functions, two of them being classical ones. This problem is encountered while studying the propagation of light in strongly scattering media such as stellar atmospheres.
\end{abstract}

1. Introduction. The integral form of the equation describing the radiative transfer of energy in a static, plane-parallel stellar atmosphere is $[\mathbf{1}]$

$$
S(a, b, \tau)=S_{0}(a, b, \tau)+a \int_{0}^{b} K\left(\tau-\tau^{\prime}\right) S\left(a, b, \tau^{\prime}\right) d \tau^{\prime},
$$

where $S$ is the source function of the radiation field and $S_{0}$ describes the radiation of the primary (internal or external) sources. These functions depend on the two parameters of the problem: the albedo $a \in] 0,1[$, which characterizes the scattering properties of the stellar plasma and the optical thickness $b>0$ of the atmosphere. They also depend on the optical depth $\tau \in[0, b]$, which is the space variable. Equation (1) means that the radiation field at level $\tau$ is the sum of the direct field from the primary sources, and the diffuse field having scattered at least once.

In the simplest scattering process conceivable, i.e., a monochromatic and isotropic one, the kernel of the integral equation (1) is the function

$$
K(\tau):=\frac{1}{2} E_{1}(|\tau|), \quad \tau \in \mathbf{R}^{*}
$$

\footnotetext{
Accepted by the editors on December 19, 2004.

Key words and phrases. Weakly singular integral equation, finite Laplace transform, sectionally analytic function, radiation transfer theory, stellar atmospheres.
} 
where $E_{1}$ is the first exponential integral function

$$
E_{1}(\tau):=\int_{0}^{1} \exp (-\tau / x) \frac{d x}{x}, \quad \tau>0 .
$$

Since $E_{1}(|\tau|) \sim-\ln (|\tau|)$ when $|\tau| \rightarrow 0^{+}$, the kernel of the integral equation (1) is weakly singular on its diagonal. The free term $S_{0}$ includes the thermal emission of the stellar plasma - of the form $(1-a) B^{*}(\tau)$, where $B^{*}$ is a known function - and the contribution $a J_{0}^{\text {ext }}(b, \tau)$ of the external sources via the boundary conditions, see the introduction of [1]. Hence, $S_{0}(a, b, \tau)=(1-a) B^{*}(\tau)+a J_{0}^{\text {ext }}(b, \tau)$. In a homogeneous and isothermal atmosphere assumed to be in local thermodynamic equilibrium, the function $B^{*}$ coincides with the Planck function $B(T)$ at the (constant) temperature $T$. Moreover, $J_{0}^{\text {ext }}=0$ in the absence of external sources and thus

$$
S_{0}(a, b, \tau)=(1-a) B(T)
$$

which shows that $S_{0}$ is independent of $\tau$ in this model. The solution $S$ to the problem (1) is then

$$
S(a, b, \tau)=(1-a) B(T) Q(a, b, \tau),
$$

where $Q$ solves the following integral equation:

$$
Q(a, b, \tau)=1+a \int_{0}^{b} K\left(\tau-\tau^{\prime}\right) Q\left(a, b, \tau^{\prime}\right) d \tau^{\prime}
$$

It is proved in $[\mathbf{2}]$ that the space $C^{0}([0, b])$ of the continuous functions from $[0, b]$ to $\mathbf{R}$ is invariant under the operator

$$
\Lambda: f \rightarrow \Lambda f(\tau):=\int_{0}^{b} K\left(\tau-\tau^{\prime}\right) f\left(\tau^{\prime}\right) d \tau^{\prime}
$$

with norm

$$
\|\Lambda\|_{\infty}=\int_{0}^{b / 2} E_{1}(\tau) d \tau=1-E_{2}(b / 2)
$$


Here, $E_{2}(\tau):=\int_{0}^{1} \exp (-\tau / x) d x$ is the exponential integral function of order 2. Equation (6), which can be written in the form

$$
Q=1+a \Lambda Q,
$$

has therefore a unique solution in $C^{0}([0, b])$ provided that $a<1$ or $b<+\infty$.

This problem is a basic one in stellar atmospheres theory, and more generally in transport theory. It describes in the simplest way the multiple scattering of some type of particles (here photons) on scattering centers distributed uniformly in a slab of finite thickness, a very simple 1D-configuration. Its applications in astrophysics and neutronics, among other fields, are presented in [9]. It is important to solve this problem very accurately, thus providing a benchmark to validate the numerical solutions of integral equations of the form (1).

Physicists and astrophysicists have developed many methods for solving integral equations of the form (1) with a convolution kernel defined by (2) [10]. The main steps for solving the prototype equation (6) are summarized in a recent article $[6]$, which contains accurate tables of the function $(1-a) Q$ for different values of the parameters $a$ and $b$. While reading this paper, one is struck by the complexity of the "classical" solution to equation (6). It requires the introduction of many intricate auxiliary functions introduced in the literature over more than 30 years. The reader quickly loses the thread of the solution, which reduces his chances of exploiting it for solving problems of the more general form (1).

The aim of the present article is to get around this difficulty by solving equation (6) straightforwardly, introducing as few auxiliary functions as possible to express its solution. These functions are briefly studied in Appendix A. The method is based on the finite Laplace transform, which reduces the problem (6) to solving a Cauchy integral equation over $[-1,+1]$, which in turn can be transformed into two Fredholm integral equations over $[0,1]$. This approach has been developed in transport theory after the publication in 1953 of the first English translation of Muskhelishvili's monograph, Singular Integral Equations [8]; see, e.g., $[\mathbf{3}, \mathbf{5}, \mathbf{7}]$. It can be considered as an extension of the Wiener-Hopf method [4] for solving integral equations of the form (1) with $b<\infty$. Both methods are characterized by an 
intensive use of the theory of (sectionally) analytic functions, which allows solution of equation (1) in a concise manner. This is obvious when comparing the solution we derive here to the classical solution of the particular problem (6), which does not use any calculation in the complex plane. The former method clarifies the origin and the role of the auxiliary functions expressing the solution to a problem of the form (1). Since these functions are independent of the source term $S_{0}$, they are "universal" for a given scattering kernel.

The remainder of this article is organized as follows. In Section 2, the finite Laplace transform of the $Q$-function is calculated on the basis of some recent developments on Cauchy integral equations [11-13]. Then the Laplace transform is inverted and the solution achieved in [6] is concisely retrieved with the help of the theorem of residues, Section 3. It involves two functions $F_{+}$and $F_{-}$with remarkable properties, as shown in Appendix B. The difficulties arising from the numerical evaluation of the latter functions are investigated in $[\mathbf{6}]$.

\section{The calculation of the finite Laplace transform of the} $Q$-function. Supposing $0<b<\infty$, we plan to solve equation (9) by Laplace transform (LT) over $[0, b]$. This operator is defined on $C^{0}([0, b])$ by

$$
\mathfrak{L} f(z):=\int_{0}^{b} f(\tau) \exp (-\tau z) d \tau, \quad z \in \mathbf{C} .
$$

Since $b<\infty$, the finite LT of a continuous function is defined and analytic in the whole complex plane. The inversion formula

$$
f(\tau)=\frac{1}{2 i \pi} \int_{c-i \infty}^{c+i \infty} \mathfrak{L} f(z) \exp (\tau z) d z
$$

is valid at any $\tau \in] 0, b[$, with no restriction on $c \in \mathbf{R}$. The symbol $f$ on the righthand side of equation (11) means that the integral is a Cauchy principal value at infinity, i.e.,

$$
f_{c-i \infty}^{c+i \infty}=\lim _{X \rightarrow+\infty} \int_{c-i X}^{c+i X} .
$$


With the intention of taking the LT of both members of equation (9), we note that the LT of the function $K$, as defined by equations (2)-(3), exists on $\mathbf{C} \backslash\{ \pm 1\}$ and is

$$
\begin{aligned}
& \mathfrak{L} K(z) \\
& \quad=w(1 / z)-\frac{1}{2} \int_{0}^{1} \frac{d u}{1-z u}-\exp (-b z) \frac{1}{2} \int_{0}^{1} \exp (-b / u) \frac{d u}{1+z u},
\end{aligned}
$$

where $w: \mathbf{C} \backslash\{ \pm 1\} \rightarrow \mathbf{C}$ denotes the function

$$
w(z):=\frac{z}{2} \int_{-1}^{+1} \frac{d u}{u+z} .
$$

The three integrals on the righthand side of equations (12)-(13) are Cauchy principal values over $] 1,+\infty[]-,\infty,-1[$ and $]-1,+1[$, respectively. From the definition ( 7 ) of the $\Lambda$-operator, we infer that the finite LT of $\Lambda f$ is

$$
\begin{aligned}
\mathfrak{L}(\Lambda f)(z)= & w(1 / z) \mathfrak{L} f(z)-\frac{1}{2} \int_{0}^{1} \mathfrak{L} f(1 / u) \frac{d u}{1-z u} \\
& -\exp (-b z) \frac{1}{2} \int_{0}^{1} \mathfrak{L} f(-1 / u) \exp (-b / u) \frac{d u}{1+z u} .
\end{aligned}
$$

In addition, the finite $\mathrm{LT}$ of the unit function is $z \rightarrow(1 / z)[1-\exp (-b z)]$. Taking the LT of both members of equation (9) and changing $z$ into $1 / z$, we obtain the following integral equation for $\mathfrak{L} Q$ :

$$
\begin{aligned}
& T(a, z) \mathfrak{L} Q(a, b, 1 / z)-\frac{a}{2} z \int_{0}^{1} \mathfrak{L} Q(a, b, 1 / u) \frac{d u}{u-z} \\
& +\exp (-b / z) \frac{a}{2} z \int_{0}^{1} \mathfrak{L} Q(a, b,-1 / u) \exp (-b / u) \frac{d u}{u+z}=c_{0}(z),
\end{aligned}
$$

where

$$
T(a, z):=1-a w(z)=1-\frac{a}{2} z \int_{-1}^{+1} \frac{d u}{u+z}
$$

and

$$
c_{0}(z):=z[1-\exp (-b / z)]
$$


The function $T$ is our first basic auxiliary function. Its main properties are summarized in Appendix A. The function $c_{0}$ satisfies the three following conditions: (i) it is defined and analytic in $\mathbf{C}^{*}$, (ii) it is bounded at infinity (with limit equal to $b$ ) and (iii) it satisfies, in a neighborhood of 0 , the conditions

$$
\lim _{\substack{z \rightarrow 0 \\ \operatorname{Re}(z)>0}} c_{0}(z)<\infty, \quad \lim _{\substack{z \rightarrow 0 \\ \operatorname{Re}(z)>0}}\left[c_{0}(-z) \exp (-b / z)\right]<\infty .
$$

Replacing $u$ by $-u$ in the second integral of equation (15), we obtain a Cauchy integral equation on $[-1,+1]$ for the function $z \rightarrow$ $\mathfrak{L} Q(a, b, 1 / z)$. Since the latter is not Hölder-continuous at 0 , it seems inappropriate to undertake the resolution of this equation. A better approach consists in observing that the functions $z \rightarrow \mathfrak{L} Q(a, b, 1 / z)$ and $z \rightarrow \mathfrak{L} Q(a, b,-1 / z) \times \exp (-b / z)$ are solutions of two coupled Cauchy integral equations on $[0,1]$, which can be uncoupled by adding and subtracting them. The resulting Cauchy integral equations are then reduced to two Fredholm integral equations of the second kind on $[0,1]$ with regular kernels. This general approach was first introduced in transfer theory by Busbridge $[\mathbf{3}]$ and developed by Mullikin et al. $[\mathbf{5}, \mathbf{7}]$ and Rutily et al. [13]. The last mentioned reference gives a synthesis that the reader may consult for details. It contains the proof of the following result, which we omit here: the unique solution, analytic in $\mathbf{C}^{*}$, to an integral equation of the form (15) with free term satisfying the conditions (i)-(iii), can be written in the form

$$
\mathfrak{L} Q(a, b, 1 / z)=\frac{1}{2}\left[u_{-}(a, b, z) \eta_{0,+}(a, b, z)+u_{+}(a, b, z) \eta_{0_{--}}(a, b, z)\right],
$$

where, for any $z \in \mathbf{C} \backslash i \mathbf{R}$,

$$
\begin{aligned}
u_{ \pm}(a, b, z):= & \mathrm{Y}[\Re(z)] H(a, z) \zeta_{ \pm}(a, b,-z) \\
& \mp \mathrm{Y}[-\Re(z)] H(a,-z) \zeta_{ \pm}(a, b, z) \exp (-b / z),
\end{aligned}
$$

and

$$
\begin{aligned}
& \eta_{0, \pm}(a, b, z):=\frac{1}{2} c_{0, \mp}(a, b,+0) \\
& \quad+\frac{z}{2 i \pi} \int_{-i \infty}^{+i \infty} H\left(a, 1 / z^{\prime}\right)\left(\zeta_{ \pm}\right)^{-}\left(a, b,-1 / z^{\prime}\right) c_{0}\left(-1 / z^{\prime}\right) \frac{d z^{\prime}}{1+z z^{\prime}}
\end{aligned}
$$


Equation (20) involves the Heaviside function $\mathrm{Y}$, which vanishes over $\mathbf{R}_{-}^{*}$ and is equal to unity over $\mathbf{R}_{+}^{*}$. On the righthand side of equation (21), we have introduced

$$
c_{0, \mp}(a, b,+0):=\lim _{\substack{z \rightarrow 0 \\ \operatorname{Re}(z)>0}}\left[c_{0}(z) \pm c_{0}(-z) \exp (-b / z)\right] .
$$

Since this limit vanishes for the particular $c_{0}(z)$ given by (17), equation (21) can be rewritten as

$$
\begin{aligned}
& \eta_{0, \pm}(a, b, z) \\
& =-\frac{1}{2 i \pi} \int_{-i \infty}^{+i \infty} H\left(a, 1 / z^{\prime}\right)\left(\zeta_{ \pm}\right)^{-}\left(a, b,-1 / z^{\prime}\right)\left[1-\exp \left(b z^{\prime}\right)\right] \frac{d z^{\prime}}{z^{\prime}\left(z^{\prime}+1 / z\right)} .
\end{aligned}
$$

The functions $H(a, z), \zeta_{+}(a, b, z)$ and $\zeta_{-}(a, b, z)$ appearing in equations (20) and (23) are the other three auxiliary functions of our approach. They are defined in Appendix A, which synthesizes their main properties. The $H$-function is a classical function of radiation transfer theory, recently reviewed in [12]. Most results concerning the functions $\zeta_{ \pm}$are summarized in [13]. They are sectionally analytic in the complex plane cut along the imaginary axis, with limits $\left(\zeta_{ \pm}\right)^{+}$and $\left(\zeta_{ \pm}\right)^{-}$on the right and lefthand sides of this axis, respectively. These limits satisfy the relation

$$
\begin{aligned}
& H\left(a, z_{0}\right)\left(\zeta_{ \pm}\right)^{-}\left(a, b,-z_{0}\right) \\
& \quad=\mp H\left(a,-z_{0}\right)\left(\zeta_{ \pm}\right)^{-}\left(a, b, z_{0}\right) \exp \left(-b / z_{0}\right)
\end{aligned}
$$

for any $z_{0} \in i \mathbf{R}^{*}$. This can be seen by putting $z \rightarrow z_{0}$ on the right, then on the left, into equation (20), taking into account the continuity of the functions $u_{ \pm}$on $i \mathbf{R}[\mathbf{1 3}]$. The limits of the functions $\zeta_{ \pm}$on both sides of the imaginary axis also satisfy the relation

$$
\begin{aligned}
H\left(a, z_{0}\right)\left(\zeta_{ \pm}\right)^{+}\left(a, b,-z_{0}\right)= & H\left(a, z_{0}\right)\left(\zeta_{ \pm}\right)^{-}\left(a, b,-z_{0}\right) \\
& \pm H\left(a,-z_{0}\right)\left(\zeta_{ \pm}\right)^{+}\left(a, b, z_{0}\right) \exp \left(-b / z_{0}\right)
\end{aligned}
$$

which follows from (A11) and Plemelj's formulae [8].

In the expression (23) of the functions $\eta_{0, \pm}$, replace the term containing $\exp \left(b z^{\prime}\right)$ by its expression given by $(24)$, with $z_{0}=-1 / z^{\prime}$. One 


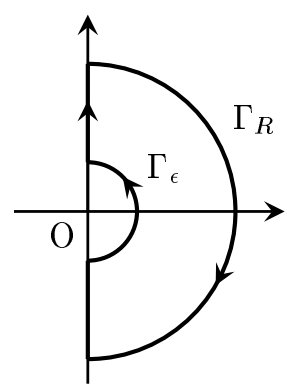

FIGURE 1. Contour for the calculation of $\eta_{0, \pm}(a, b, z)$.

obtains

$$
=-\frac{1}{2 i \pi} \int_{-i \infty}^{+i \infty} H\left(a, 1 / z^{\prime}\right)\left(\zeta_{ \pm}\right)^{-}\left(a, b,-1 / z^{\prime}\right)\left[\frac{1}{z^{\prime}+1 / z} \pm \frac{1}{z^{\prime}-1 / z}\right] \frac{d z^{\prime}}{z^{\prime}} .
$$

This integral may be calculated using the contour of Figure 1. The integral along the half-circle $\Gamma_{\varepsilon}$ tends to $-(1 / 2) H(a, \infty) \zeta_{ \pm}(a, b,-\infty)(z \mp$ $z)$ as $\varepsilon \rightarrow 0$, where $H(a, \infty)$ is the limit of $H(a, z)$ when $z \rightarrow \infty$ in any part of the complex plane. This limit is given by equation (A9). $\zeta_{ \pm}(a, b,-\infty)$ denotes the limit of $\zeta_{ \pm}(a, b, z)$ when $z \rightarrow \infty$ in the left complex half-plane. In equation (A18), this limit is expressed in terms of $\zeta_{ \pm}(a, b,+\infty)$. The integral along $\Gamma_{R}$ tends to 0 when $R \rightarrow \infty$. From the theorem of residues, we have for any $z \in \mathbf{C} \backslash i \mathbf{R}$,

$$
\begin{aligned}
& \eta_{0, \pm}(a, b, z)= \frac{z}{\sqrt{1-a}}(1 \mp 1)\left(\zeta_{ \pm}\right)(a, b,+\infty) \\
&-z\left\{\mathrm{Y}[-\Re(z)] H(a,-z) \zeta_{ \pm}(a, b, z)\right. \\
&\left.\mp \mathrm{Y}[\Re(z)] H(a, z) \zeta_{ \pm}(a, b,-z)\right\} .
\end{aligned}
$$

The end of the calculation of $\mathfrak{L} Q$ follows from equations (19), (20) and (27). Changing $z$ into $1 / z$, we get

$$
\mathfrak{L} Q(a, b, z)=\frac{1}{\sqrt{1-a}} \frac{1}{z} \zeta_{-}(a, b,+\infty) u_{+}(a, b, 1 / z)
$$


a result valid in principle on $\mathbf{C} \backslash i \mathbf{R}$. In fact, it can easily be seen that the limits of this function on both sides of the imaginary axis coincide. This makes it possible to extend the relation (28) by continuity on $i \mathbf{R}$, including 0 . Such an extension will not be used subsequently.

3. Inverting the Laplace transform. The inversion formula (11) reads here, for any $\tau \in] 0, b[$,

$$
Q(a, b, \tau)=\frac{1}{2 i \pi} \int_{c-i \infty}^{c+i \infty} \mathfrak{L} Q(a, b, z) \exp (\tau z) d z,
$$

$c \neq 0$. Substituting (28) in this expression and replacing $u_{+}(a, b, 1 / z)$ by its definition (20), we obtain

(30) $Q(a, b, \tau)$

$$
=\frac{1}{\sqrt{1-a}} \zeta_{-}(a, b,+\infty) \frac{1}{2 i \pi} \int_{c^{\prime}-i \infty}^{c^{\prime}+i \infty} H(a, 1 / z) \zeta_{+}(a, b,-1 / z) \exp (\tau z) \frac{d z}{z},
$$

where $c^{\prime}=|c|>0$. The integral on the righthand side can be calculated with the help of the residue theorem applied to a contour in the left half-plane, owing to the presence of the exponential. A difficulty arises because $c^{\prime}>0$ and the imaginary axis should not be crossed since the functions $\zeta_{ \pm}$are discontinuous on this axis. Nevertheless, one can derive the identity

$$
\begin{aligned}
\frac{1}{2 i \pi} \int_{c^{\prime}-i \infty}^{c^{\prime}+i \infty} & H(a, 1 / z) \zeta_{+}(a, b,-1 / z) \exp (\tau z) \frac{d z}{z} \\
= & \frac{1}{2 i \pi} \int_{-i \infty}^{+i \infty} H(a, 1 / z)\left(\zeta_{+}\right)^{-}(a, b,-1 / z) \exp (\tau z) \frac{d z}{z}
\end{aligned}
$$

from the contour of Figure 2(a), since the integral on the half-circle $\Gamma_{\varepsilon}$ tends to 0 when $\varepsilon \rightarrow 0$, due to equation (A18). The last integral is a Cauchy principal value at 0 . Then the limits of the functions $\zeta_{ \pm}$on the lefthand side of the imaginary axis are expressed in terms of their limits on the righthand side with the help of (25), which shows that

$$
\begin{aligned}
& \frac{1}{2 i \pi} \int_{-i \infty}^{+i \infty} H(a, 1 / z)\left(\zeta_{+}\right)^{-}(a, b,-1 / z) \exp (\tau z) \frac{d z}{z} \\
= & \frac{1}{2 i \pi} \int_{-i \infty}^{+i \infty} H(a, 1 / z)\left(\zeta_{+}\right)^{+}(a, b,-1 / z)[\exp (\tau z)+\exp ((b-\tau) z)] \frac{d z}{z}
\end{aligned}
$$



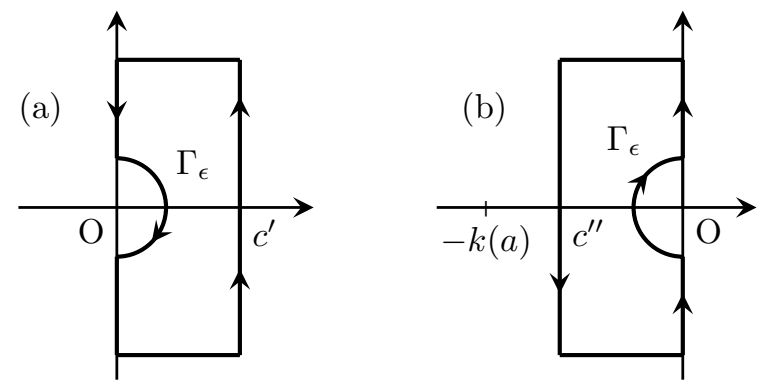

FIGURE 2. Contours (a) and (b) for the proof of equations (31) and (33).

The last integral is calculated by displacing the line of integration on the left side of the imaginary axis, between the origin and the point of abscissa $-k(a)$ where the function $z \rightarrow H(a, 1 / z)$ diverges; see the comments after equation (A8) in Appendix A, which contains the definition of $k(a)$. Applying the residue theorem to the contour of Figure 2(b) and observing that the integral on $\Gamma_{\varepsilon}$ tends to $-H(a, \infty) \zeta_{+}(a, b,+\infty)$ when $\varepsilon \rightarrow 0$, one obtains

$$
\begin{aligned}
& \frac{1}{2 i \pi} \int_{-i \infty}^{+i \infty} H(a, 1 / z)\left(\zeta_{+}\right)^{+}(a, b,-1 / z)[\exp (\tau z)+\exp ((b-\tau) z)] \frac{d z}{z} \\
& =\frac{1}{\sqrt{1-a}} \zeta_{+}(a, b,+\infty) \\
& \quad+\frac{1}{2 i \pi} \int_{c^{\prime \prime}-i \infty}^{c^{\prime \prime}+i \infty} H(a, 1 / z) \zeta_{+}(a, b,-1 / z)[\exp (\tau z)+\exp ((b-\tau) z)] \frac{d z}{z},
\end{aligned}
$$

where $\left.c^{\prime \prime} \in\right]-k(a), 0[$. For $\tau \in] 0, b[$, the last integral coincides with the function $-F_{+}(a, b, \tau)$ introduced in Appendix B, equation (B1).

According to equations (30)-(33), we finally have

$$
Q(a, b, \tau)=\frac{1}{\sqrt{1-a}} \zeta_{-}(a, b,+\infty)\left[\frac{1}{\sqrt{1-a}} \zeta_{+}(a, b,+\infty)-F_{+}(a, b, \tau)\right],
$$

or, using equation (B3)

$$
Q(a, b, \tau)=\left[1+F_{-}(a, b, 0)\right]\left[1+F_{+}(a, b, 0)-F_{+}(a, b, \tau)\right] .
$$


This expression is valid a priori over $] 0, b[$, but it can be extended by continuity over $[0, b]$, since the functions $F_{ \pm}$are continuous on the right at 0 and on the left at $b$. One has in particular

$$
Q(a, b, 0)=Q(a, b, b)=1+F_{-}(a, b, 0) .
$$

These results are similar to those of $[6]$, where details concerning the numerical evaluation of the function $Q$ can be found. Ten-figure tables of the function $(1-a) Q$ are given there. The functions $F_{ \pm}$ are computable once their definition (B1) has been transformed by the method of residues, as outlined in Appendix B.

4. Conclusion. Using the finite Laplace transform, we derived in a concise way the expression (35) of the solution to equation (6), which is appropriate for numerical evaluation [6]. Our main objective was to come directly to this expression, with emphasis on the manner in which the four auxiliary functions of the problem were generated. They are (i) the function $T=T(a, z)$, which characterizes the solution to equation (6) in an infinite medium (the range $[0, b]$ is replaced by R), (ii) the function $H=H(a, z)$ which expresses its solution in a halfspace $(b=\infty)$, and (iii) the functions $\zeta_{ \pm}=\zeta_{ \pm}(a, b, z)$ that complete the previous ones in the finite case, $b<\infty$. The main properties of these functions are summarized in Appendix A. We note that the $T$-function can be expressed in terms of elementary transcendental functions (equation (A3)), the $H$-function is defined explicitly by an integral on $[0,1]$ (equation (A10)) and the functions $\zeta_{ \pm}$are defined implicitly as the solutions to Fredholm integral equations of the second kind (equation (A13)). It seems that the problem (6) has no exact closed-form solution for $b<\infty$.

The approach of this article is appropriate for solving integral equations of the form (1), with a kernel defined by (2) and any free term. It also applies to convolution kernels defined by functions more general than (2), for example, of the form

$$
K(\tau):=\int_{I} \Psi(x) \exp (-|\tau| / x) d x,
$$

where $I$ is an interval of $\mathbf{R}$ and $\Psi$ a function ensuring the existence of the integral. This class of kernels models scattering processes more 
complex than the one considered in this article, which corresponds to $I=] 0,1]$ and $\Psi(x)=(1 / 2)(1 / x)$.

Acknowledgments. We gratefully thank Professeurs M. Ahues and A. Largillier (Université Jean Monnet, Saint-Etienne, France) for making several useful comments on this work. We also thank Professeurs J. Bergeat and G. Paturel (Observatoire de Lyon) for their careful reading of the manuscript.

\section{APPENDix}

A. The auxiliary functions associated to the problem (6). These functions are $T=T(a, z), H=H(a, z)$, and $\zeta_{ \pm}=\zeta_{ \pm}(a, b, z)$, where $z \in \mathbf{C}$.

A.1 The T-function. This function is defined by

$$
T(a, z):=1-\frac{a}{2} z \int_{-1}^{+1} \frac{d u}{u+z}, \quad z \neq \pm 1 .
$$

It describes the multiple scattering of photons in an unbounded medium for the adopted scattering law. It is defined in the whole complex plane $\mathbf{C}$, except at \pm 1 , provided that the integral is calculated in the sense of the Cauchy principal value when $z \in]-1,+1[$. It is thus sectionally analytic in $\mathbf{C} \backslash[-1,+1]$, its limits on both sides of the segment ] $-1,+1[$ being given from Plemelj formulae [8] by

$$
T^{ \pm}(a, u)=T(a, u) \pm i \pi(a / 2) u, \quad-1<u<+1 .
$$

Here, $T^{+}(a, u)$, respectively $T^{-}(a, u)$, denotes the limit of $T(a, z)$ when $z$ tends to $u \in]-1,+1[$ from above, respectively below, the real axis, and

$$
T(a, u)=1-\frac{a}{2} u \ln \left(\frac{1+u}{1-u}\right), \quad f-1<u<+1 .
$$

The roots of the characteristic equation $T(a, z)=0$ are important for solving integral equations of the form (1) with kernel defined by (2). When $0<a<1$, this equation has four nonzero roots in $\mathbf{C}$, 
namely two pairs of opposite real numbers since the $T$-function is even [4]. There is a unique root strictly greater than unity, denoted by $1 / k(a), 0<k(a)<1$, which is calculated by solving the transcendental equation

$$
T(a, 1 / k(a))=1-\frac{a}{2} \frac{1}{k(a)} \ln \left[\frac{1+k(a)}{1-k(a)}\right]=0 .
$$

Its unique solution $k(a)$ in ]0, 1 [ is given by [5]

$$
k(a)=\sqrt{1-a} \exp \left[\int_{0}^{1} \theta(a, u) \frac{d u}{u}\right],
$$

where

$$
\theta(a, u):=\frac{1}{\pi} \arg \left[T^{+}(a, u)\right], \quad 0 \leq u<1 .
$$

Here, $\arg (z)$ is the principal value of the argument of $z \in \mathbf{C}$. It can be computed using the atan2-function, $\operatorname{since} \arg (x+i y)=\operatorname{atan} 2(y, x)$.

A.2 The $H$-function. This function is the unique solution, analytic in the right complex half-plane, of the integral equation

$$
T(a, z) H(a, z)=1+\frac{a}{2} z \int_{0}^{1} H(a, u) \frac{d u}{u-z}, \quad z \neq \pm 1 .
$$

This is a Cauchy integral equation on $[0,1]$, which defines the extension of $H$ outside this interval. The function $H$ satisfies the Wiener-Hopf factorization relation

$$
T(a, z) H(a, z) H(a,-z)=1, \quad z \neq \pm 1,
$$

first proved regardless of (A7), see, e.g., [4], then as a result of (A7) [12]. We note that it implies the divergence of the $H$-function at the solutions of the equation $T(a, z)=0$ located in the left complex halfplane, especially at $z=-1 / k(a)$. The relations (A7)-(A8) also mean that the $H$-function is bounded at infinity, with limit

$$
H(a, \infty)=\frac{1}{\sqrt{1-a}}
$$


whatever the region of the complex plane where $z$ goes to infinity. This results from the fact that $T(a, \infty)=1-a$.

Many analytical expressions of the function $H$ have been derived since the introduction of this function in the thirties. The following expression is valid in the right complex half-plane [5]

$$
\begin{aligned}
& H(a, z) \\
& \quad=\frac{k(a)}{\sqrt{1-a}} \frac{1+z}{1+k(a) z} \exp \left[-z \int_{0}^{1} \theta(a, u) \frac{d u}{u+z}\right], \quad \Re(z) \geq 0,
\end{aligned}
$$

where $\theta$ is the function defined by (A6). We used it to compute $H(a, z)$ over $[0,1]$ and at $1 / k(a)$, a necessary step for computing the functions $F_{ \pm}$appearing in our final result (35), see Appendix B.

A.3 The functions $\zeta_{ \pm}$. These functions are sectionally analytic in $\mathbf{C} \backslash i \mathbf{R}$, with limits $\left(\zeta_{ \pm}\right)^{+}$and $\left(\zeta_{ \pm}\right)^{-}$on the right and lefthand sides of the imaginary axis, respectively. They are defined by the relations

$$
\begin{aligned}
& \zeta_{ \pm}(a, b, z)=1 \\
& \pm \frac{z}{2 i \pi} \int_{-i \infty}^{+i \infty} \frac{H\left(a,-1 / z^{\prime}\right)}{H\left(a, 1 / z^{\prime}\right)} \exp \left(-b z^{\prime}\right)\left(\zeta_{ \pm}\right)^{+}\left(a, b, 1 / z^{\prime}\right) \frac{d z^{\prime}}{1+z z^{\prime}}
\end{aligned}
$$

which can be transformed into Fredholm integral equations of the second kind on $[0,1]$ using the residue theorem [13]. We suppose here the existence and uniqueness of the solution to equation (A11), and refer to $[\mathbf{1 3}]$ for the proof of the relation

$$
\frac{1}{2}\left[\zeta_{+}(a, b, z) \zeta_{-}(a, b,-z)+\zeta_{-}(a, b, z) \zeta_{+}(a, b,-z)\right]=1,
$$

which is valid over $\mathbf{C} \backslash i \mathbf{R}$.

The transformation of equation (A11) into a couple of Fredholm integral equations on $[0,1]$ is described in Section V of $[\mathbf{1 3}]$. We reproduce the relation (71) of this article, valid for any $z$ in $\mathbf{C} \backslash\{]-1,0[\cup\{-1 / k(a)\}\}$

$$
\begin{aligned}
\zeta_{ \pm}(a, b, z)= & 1 \pm M_{ \pm}(a, b) \frac{2 k z}{1+k z} \\
& \mp \mathrm{Y}[-\Re(z)] \frac{1}{T(a, z)} \frac{\exp (b / z)}{H^{2}(a,-z)} \zeta_{ \pm}(a, b,-z) \\
& \pm \frac{a}{2} z \int_{0}^{1} \frac{g(a, u)}{H^{2}(a, u)} \exp (-b / u) \zeta_{ \pm}(a, b, u) \frac{d u}{u+z}
\end{aligned}
$$


We have introduced in the righthand side

$$
M_{ \pm}(a, b):=q(a, b) \zeta_{ \pm}(a, b, 1 / k(a)),
$$

where

$$
\begin{gathered}
q(a, b):=\frac{1}{2} \frac{R(a)}{H^{2}(a, 1 / k(a))} \exp (-k(a) b), \\
R(a):=\frac{k(a)}{T^{\prime}(a, 1 / k(a))}=\frac{1-k^{2}(a)}{k^{2}(a)+a-1},
\end{gathered}
$$

and the function $g$ in the integral term is

$$
\begin{aligned}
g(a, u) & :=\frac{1}{T^{+}(a, u) T^{-}(a, u)} \\
& =\frac{1}{T^{2}(a, u)+(\pi a u / 2)^{2}}, \quad 0 \leq u<1 .
\end{aligned}
$$

Let $z$ go to infinity in both members of equation (A13), first in the left complex half-plane, then in the right one. Writing $\zeta_{ \pm}(a, b,-\infty)$ and $\zeta_{ \pm}(a, b,+\infty)$ the limits obtained in this way, we have

$$
\zeta_{ \pm}(a, b,-\infty)=(1 \mp 1) \zeta_{ \pm}(a, b,+\infty) .
$$

Consequently,

$$
\zeta_{-}(a, b,+\infty) \zeta_{+}(a, b,+\infty)=1,
$$

due to (A12).

The numerical calculation of the functions $\zeta_{ \pm}$is carried out starting from equation (A13), which consists of two Fredholm integral equations on $[0,1]$. A difficulty arises because the coefficients $M_{ \pm}(a, b)$ contain the unknown values of $\zeta_{ \pm}$at $1 / k(a)$. It is overcome by putting $z=1 / k(a)$ into equation (A13) to calculate $M_{ \pm}(a, b)$, which is next substituted into the righthand side of (A13). The expression of the functions $\zeta_{ \pm}$in the right half-plane becomes [13]

$$
\zeta_{ \pm}(a, b, z)=\rho_{ \pm}(a, b, z) \pm M_{ \pm}(a, b) \sigma_{ \pm}(a, b, z), \quad \Re(z) \geq 0,
$$


where $\rho_{ \pm}$and $\sigma_{ \pm}$are the solutions to the integral equations

$$
\begin{aligned}
\rho_{ \pm}(a, b, z)= & 1 \pm \frac{a}{2} z \int_{0}^{1} \frac{g(a, u)}{H^{2}(a, u)} \exp (-b / u) \rho_{ \pm}(a, b, u) \frac{d u}{u+z} \\
\sigma_{ \pm}(a, b, z)= & \frac{2 k(a) z}{1+k(a) z} \\
& \pm \frac{a}{2} z \int_{0}^{1} \frac{g(a, u)}{H^{2}(a, u)} \exp (-b / u) \sigma_{ \pm}(a, b, u) \frac{d u}{u+z}
\end{aligned}
$$

Once they have been solved on $[0,1]$, these equations define the functions $z \rightarrow \rho_{ \pm}(a, b, z)$ and $z \rightarrow \sigma_{ \pm}(a, b, z)$ everywhere in the right complex half-plane, in particular at $z=1 / k(a)$. Whence the coefficients $M_{ \pm}(a, b)$ from equations (A14) and (A20) are

$$
M_{ \pm}(a, b)=\frac{q(a, b) \rho_{ \pm}(a, b, 1 / k(a))}{1 \mp q(a, b) \sigma_{ \pm}(a, b, 1 / k(a))}
$$

$q(a, b)$ being defined by (A15)-(A16).

Calculating the functions $\zeta_{ \pm}$thus requires the numerical solution of equations (A21)-(A22). In fact, it can be proved that the solution of equations (A22) comes down to that of equations (A21). This means that the functions $\sigma_{ \pm}$are analytically expressible in terms of the functions $\rho_{ \pm}$. It follows that only equations (A21) have to be solved numerically in view of computing the functions $\zeta_{ \pm}$. The details are not given here, since there is no inconvenience, at the level of accuracy, to solve both sets of equations numerically. This results from the regularity of the free terms and kernels of equations (A21)-(A22) on $[0,1]$ and $[0,1] \times[0,1]$, respectively. Their solutions $\rho_{ \pm}$and $\sigma_{ \pm}$are easily computable since they are regular and smooth on $[0,1]$, i.e., not only continuous, but differentiable everywhere in $[0,1]$, including at 0 . We plan to publish ten-figure tables of these functions in the future.

B. The functions $F_{ \pm}=F_{ \pm}(a, b, \tau)$. These functions are defined by the relation

$$
\begin{aligned}
& F_{ \pm}(a, b, \tau):=-\frac{1}{2}[\delta(\tau) \pm \delta(b-\tau)] \\
- & \frac{1}{2 i \pi} \int_{c-i \infty}^{c+i \infty} H(a, 1 / z) \zeta_{ \pm}(a, b,-1 / z)[\exp (\tau z) \pm \exp ((b-\tau) z)] \frac{d z}{z}
\end{aligned}
$$


where $-k(a)<c<0 . \delta(\tau)$ and $\delta(b-\tau)$ are the Dirac distributions at 0 and $b$, respectively.

B.1 Three properties of the functions $F_{ \pm}$. We first prove the following relations:

$$
\begin{gathered}
F_{ \pm}(a, b, b-\tau)= \pm F_{ \pm}(a, b, \tau), \\
F_{ \pm}(a, b, 0)=\frac{1}{\sqrt{1-a}} \zeta_{ \pm}(a, b,+\infty)-1, \\
(1-a)\left[1+F_{+}(a, b, 0)\right]\left[1+F_{-}(a, b, 0)\right]=1 .
\end{gathered}
$$

Equation (B2) is evident and equation (B4) is a direct consequence of equations (B3) and (A19). To derive (B3), we note that

$$
\begin{aligned}
& F_{ \pm}(a, b, 0) \\
& \quad=-\frac{1}{2}-\frac{1}{2 i \pi} \int_{c-i \infty}^{c+i \infty} H(a, 1 / z) \zeta_{ \pm}(a, b,-1 / z)[1 \pm \exp (b z)] \frac{d z}{z}
\end{aligned}
$$

The integral is transformed with the help of the residue theorem applied to the contour of Figure 3, within which the integrand is analytic. Since the integral along the half-circle $\Gamma_{\varepsilon}$ tends to $(1 / 2) H(a, \infty) \zeta_{ \pm}$ $(a, b+\infty)(1 \pm 1)$ when $\varepsilon \rightarrow 0$, we obtain

$$
\begin{aligned}
& F_{ \pm}(a, b, 0) \\
& =-\frac{1}{2}+\frac{1}{2}(1 \pm 1) H(a, \infty) \zeta_{ \pm}(a, b,+\infty) \\
& \quad-\frac{1}{2 i \pi} \int_{-i \infty}^{+i \infty} H(a, 1 / z)\left(\zeta_{ \pm}\right)^{+}(a, b,-1 / z)[1 \pm \exp (b z)] \frac{d z}{z} .
\end{aligned}
$$

Put

$$
\begin{aligned}
& (\alpha)=-\frac{1}{2 i \pi} \int_{-i \infty}^{+i \infty} H(a, 1 / z)\left(\zeta_{ \pm}\right)^{+}(a, b,-1 / z) \frac{d z}{z}, \\
& (\beta)=\mp \frac{1}{2 i \pi} \int_{-i \infty}^{+i \infty} H(a, 1 / z)\left(\zeta_{ \pm}\right)^{+}(a, b,-1 / z) \exp (b z) \frac{d z}{z},
\end{aligned}
$$




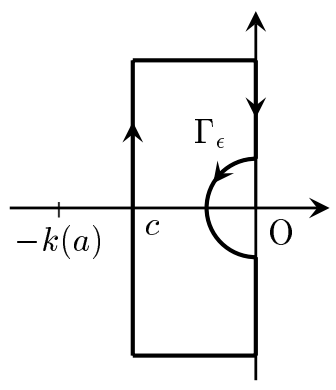

FIGURE 3. Contour for the calculation of $F_{ \pm}(a, b, 0)$.

so that the integral term in $(\mathrm{B} 6)$ is $(\alpha)+(\beta)$. From equation $(25)$ with $z_{0}=1 / z$, we have

$$
\begin{aligned}
(\beta)= & -\frac{1}{2 i \pi} \int_{-i \infty}^{+i \infty} H(a,-1 / z)\left(\zeta_{ \pm}\right)^{+}(a, b, 1 / z) \frac{d z}{z} \\
& +\frac{1}{2 i \pi} \int_{-i \infty}^{+i \infty} H(a,-1 / z)\left(\zeta_{ \pm}\right)^{-}(a, b, 1 / z) \frac{d z}{z} \\
= & +\frac{1}{2 i \pi} \int_{-i \infty}^{+i \infty} H(a, 1 / z)\left(\zeta_{ \pm}\right)^{+}(a, b,-1 / z) \frac{d z}{z} \\
& +\frac{1}{2 i \pi} \int_{-i \infty}^{+i \infty} H(a,-1 / z)\left(\zeta_{ \pm}\right)^{-}(a, b, 1 / z) \frac{d z}{z}
\end{aligned}
$$

Therefore

$$
(\alpha)+(\beta)=+\frac{1}{2 i \pi} \int_{-i \infty}^{+i \infty} H(a,-1 / z)\left(\zeta_{ \pm}\right)^{-}(a, b, 1 / z) \frac{d z}{z} .
$$

Now integrate the function $z \rightarrow H(a,-1 / z) \zeta_{ \pm}(a, b, 1 / z) / z$ along the contour of Figure 4 . When $\varepsilon \rightarrow 0$, the integral over the halfcircle $\Gamma_{\varepsilon}$ tends to $-(1 / 2) H(a, \infty) \zeta_{ \pm}(a, b,-\infty)=-(1 / 2) H(a, \infty)(1 \mp 1)$ $\zeta_{ \pm}(a, b,+\infty)$ due to equation (A18), and the integral over $\Gamma_{R}$ tends to $1 / 2$ when $R$ goes to infinity. Hence

$$
(\alpha)+(\beta)=\frac{1}{2}(1 \mp 1) H(a, \infty) \zeta_{ \pm}(a, b,+\infty)-\frac{1}{2},
$$

and equation (B6) leads to (B3) since $H(a, \infty)=1 / \sqrt{1-a}$ from equation (A9). 


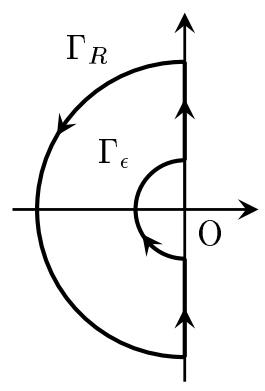

FIGURE 4. Contour for the calculation of $(\alpha)+(\beta)$.

B.2 Numerical calculation of $F_{ \pm}(a, b, \tau)$. It is carried out by replacing $H(a, 1 / z)$ by $1 /[T(a, 1 / z) H(a,-1 / z)]$ in the integral of (B1), according to (A8). This reveals the pole $z=-k(a)$ of the integrand in the left complex half-plane, together with the cut $]-\infty,-1]$ insuring that the function $z \rightarrow T(a, 1 / z)$ is single-valued in this half-plane. This function is then sectionally analytic in the complex plane cut along ]$-\infty,-1[\cup]+1,+\infty\left[\right.$, with limits $T^{ \pm}(a, 1 / u)$ on the cut given by (A2). Next, the residue theorem is applied to the contour of Figure 5, which passes round the cut. The unique pole within the contour is $z=-k(a)$, with residue

$[R(a) / H(a, 1 / k(a))] \zeta_{ \pm}(a, b, 1 / k(a))[\exp (-k(a) \tau) \pm \exp (-k(a)(b-\tau))]$,

since $R(a)$ is defined by (A16). The integral on the half-circle $\Gamma_{R}$ tends to $[\delta(\tau) \pm \delta(b-\tau)] / 2$ when $R \rightarrow \infty$, the integral on $\Gamma_{-1}$ tends to 0 with the radius, and the limit of the integral over $A B+C D$ is

$$
\frac{a}{2} \int_{1}^{+\infty} \frac{g(a, 1 / v)}{H(a, 1 / v)} \zeta_{ \pm}(a, b, 1 / v)[\exp (-\tau v) \pm \exp (-(b-\tau) v)] \frac{d v}{v^{2}},
$$

where $g$ is defined by equation (A17). Putting $u=1 / v$ in the above integral, one obtains

$$
\begin{aligned}
= & \frac{R(a)}{H(a, 1 / k(a))} \zeta_{ \pm}(a, b, 1 / k(a))[\exp (-k(a) \tau) \pm \exp (-k(a)(b-\tau))] \\
& +\frac{a}{2} \int_{0}^{1} \frac{g(a, u)}{H(a, u)} \zeta_{ \pm}(a, b, u)[\exp (-\tau / u) \pm \exp (-(b-\tau) / u)] d u .
\end{aligned}
$$




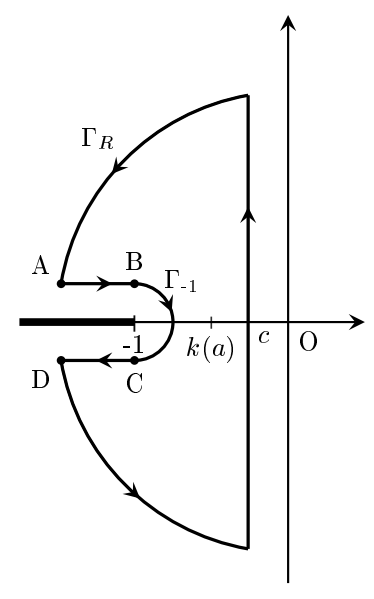

FIGURE 5. Contour for the calculation of the functions $F_{ \pm}$.

For given functions $k(a), H(a, u)$ and $\zeta_{ \pm}(a, b, u)$, the numerical evaluation of the functions $F_{ \pm}$by means of this expression is easy, except when $a \rightarrow 1$ as regards the integrated term and when $\tau \rightarrow 0^{+}$or $b^{-}$as regards the integral term. These difficulties are overcome in $[6]$.

\section{REFERENCES}

1. M. Ahues, F. D'Almeida, A. Largillier, O. Titaud and P. Vasconcelos, An $L^{1}$ refined projection approximate solution of the radiation transfer equation in stellar atmospheres, J. Comput. Appl. Math. 140 (2002), 13-26.

2. M. Ahues, A. Largillier and O. Titaud, The roles of weak singularity and the grid uniformity in relative error bounds, Numer. Funct. Anal. Optim. 22 (2002), 789-814.

3. I.W. Busbridge, On the $X$ - and $Y$-functions of $S$. Chandrasekhar, Astrophys. J. 122 (1955), 327-348.

4. - The mathematics of radiative transfer, Cambridge University Press, London, 1960.

5. J.L. Carlstedt and T.W. Mullikin, Chandrasekhar $X$ - and $Y$-functions, Astrophys. J. Suppl. 12 (1966), 449-585.

6. L. Chevallier and B. Rutily, Exact solution of the standard transfer problem in a stellar atmosphere, J. Quantit. Spectro. Radiative Transfer 91 (2005), 373-391.

7. T.W. Mullikin, Chandrasekhar's $X$ and $Y$ equations, Trans. Amer. Math. Soc. 113 (1964), 316-332. 
8. N.I. Muskhelishvili, Singular integral equations, OGIZ, Moscow-Leningrad, 1946; Noordhoff, Groningen, Holland, 1953; Dover, New York, 1992.

9. B. Rutily, Multiple scattering theory and integral equations, in Integral methods in science and engineering (C. Constanda, M. Ahues and A. Largillier, eds.), Birkhäuser, Boston, 2004.

10. B. Rutily and J. Bergeat, The solution of the Schwarzschild-Milne integral equation in an homogeneous isotropically scattering plane-parallel medium, J. Quant. Spectro. Radiative Transfer 51 (1994), 823-847.

11. Radiative transfer in plane-parallel media and Cauchy integral equations, I. The N-function, Transport Theory Statist. Phys. 31 (2002), 659-671.

12. B. Rutily, J. Bergeat and L. Chevallier, Radiative transfer in plane-parallel media and Cauchy integral equations, II. The H-function, submitted.

13. B. Rutily, L. Chevallier and J. Bergeat, Radiative transfer in plane-parallel media and Cauchy integral equations, III. The finite case, submitted.

Centre de Recherche Astronomique de Lyon, (UMr 5574 du CNRS), Observatoire de Lyon, 9 avenue Charles André, 69561 Saint-Genis-Laval Cedex, France

E-mail address: rutily@obs.univ-lyon1.fr

Centre de Recherche Astronomique de Lyon, (UMR 5574 du CNRS), Observatoire de Lyon, 9 avenue Charles André, 69561 Saint-Genis-Laval Cedex, France

E-mail address: loic.chevallier@obs.univ-lyon1.fr 